\title{
ON MEASURES OF ANALYTIC TYPE
}

\author{
LOUIS PIGNO AND BRENT SMITH
}

\begin{abstract}
The purpose of this note is to show how the characterization of an absolutely continuous or singular measure of $\mathbf{R}$. Doss can be used to prove the Helson-Lowdenslager Theorem for measures of analytic type. Our methods were inspired by the work of P. Cohen and H. Davenport on the $L^{1}$ norm of exponential sums.
\end{abstract}

Let $G$ be an infinite LCA group with character group $\Gamma$ and $M(G)$ the customary convolution algebra of bounded Borel measures on $G$; let ' denote the FourierStieltjes transform. We say $\Gamma$ is totally ordered if $\Gamma$ is discrete and there exists a semigroup $P$ such that $(P) \cap(-P)=\{0\}$ and $(P) \cup(-P)=\Gamma$. A measure $\mu \in M(G)$ is said to be of analytic type if $\hat{\mu}(\gamma)=0$ for all $\gamma \in \Gamma \backslash P=\{\gamma \in \Gamma$ : $\gamma<0\}$.

For $\mu \in M(G)$ put $\mu=\mu_{a}+\mu_{s}$ where $\mu_{a}$ is absolutely continuous with respect to Haar measure on $G$ and $\mu_{s}$ is singular with respect to Haar measure. Let $M_{a}(G)$ and $M_{s}(G)$ denote the space of absolutely continuous and singular measures, respectively. For any. $K \subset \Gamma$, let $A_{K}$ be the set of all trigonometric polynomials on $G$ of the form $p(x)=\Sigma c_{\gamma} \gamma(-x)$ such that $\|p\|_{\infty}<1$ and $\gamma \notin K$. For $\mu \in M(G)$ we define

$$
\|\mu\|_{K}=\sup _{p \in A_{K}}\left|\sum c_{\gamma} \hat{\mu}(\gamma)\right| \text { and } \lim _{K \rightarrow \infty}\|\mu\|_{K}=\inf _{K}\|\mu\|_{K}
$$

here $K$ varies through some presumably interesting family of subsets of $\Gamma$; for example $K$ can run through the compact sets.

The following quantitatively precise result can be found in [4].

Proposition 1 (R. Doss). For $\mu \in M(G)$ and $K$ compact in $\Gamma$

$$
\lim _{K \rightarrow \infty}\|\mu\|_{K}=\left\|\mu_{s}\right\|
$$

A modicum of complex variables will be needed in the sequel.

LEMMA 1. Let $T(z)=z /(z+3 a)$ where $0<a<1$. For $M \in \mathbf{Z}^{+}$put $S_{M}=\{z \in$ C: $-a<\operatorname{Re} z \leqslant 1,-M \leqslant \operatorname{Im} z \leqslant M$ \}. Then given $\delta>0$ and $M \in \mathbf{Z}^{+}$there is $a$ polynomial of the form

$$
T_{M}(z)=a_{1} z+\cdots+a_{n} z^{n}
$$

Received by the editors May 6, 1980.

1980 Mathematics Subject Classification. Primary 43A17. 
such that

(i) $\left|T(z)-T_{M}(z)\right|<\delta$ for all $z \in S_{M}$,

(ii) $\operatorname{Re}\left\{T_{M}(z)\right\} \geqslant\{1 /(1+3 a)\} \operatorname{Re} z-\delta / 2$ if $0<\operatorname{Re} z<1$ and

(iii) $\left|T_{M}(z)\right|^{2} \leqslant 1+\delta$ for all $z \in S_{M}$.

Proof. Notice that $\operatorname{Re}[T(z)]>\{1 /(1+3 a)\} \operatorname{Re} z$ if $0<\operatorname{Re} z<1$ and $T$ maps $S_{M}$ into the open unit disc. Fix any real number $r$ greater than $\left[1+M^{2}\right] / 4 a$. Then the disc $\{z \in \mathrm{C}:|z-r|<3 a+r\}$ contains $S_{M}$. Using the fact that $T(0)=0$, we expand $T$ in a power series about $r$ to obtain a polynomial of the form (\#) satisfying (i)-(iii).

LeMma 2. Let $T(z)=z /(z+3 a)(0<a<1)$. Then given $\varepsilon>0$ there is $a \xi>0$ and $a b>0$ such that if $-b \leq \operatorname{Re} z<1$ and $\operatorname{Re}\{z /(z+3 a)\}<\xi$ then $|T(z)|<$ $\varepsilon / 4$ and if $c=-b /(-b+3 a)$ then $-c /(-c+\xi)<\varepsilon$.

TheOREM 1. Let $\Gamma$ be totally ordered, $\nu \in M_{s}(G)$ and $|\nu|$ concentrated on $\Omega$. Then given $\varepsilon>0$ there is a trigonometric polynomial $k$ having only positive frequencies, $E \subset \Omega$ and $F \subset G$ such that

(a) $\|k\|_{\infty}<1+\varepsilon$

(b) $|k-1|<\varepsilon$ on $\Omega \backslash E$ and $|\nu|(E)<\varepsilon$,

(c) $|k|<\varepsilon$ on $G \backslash F$ and $m_{G}(F)<\varepsilon$.

Here $m_{G}$ is the normalized Haar measure on $G$.

Proof. Let $\nu \in M_{s}(G)$ and $|\nu|$ concentrated on $\Omega$; assume $\|\nu\|=1$. Let $0<\varepsilon<$ 1 be given; choose $\delta>0$ such that $\left(4 \delta+\delta^{2}\right)^{1 / 2}<\varepsilon$. Let $a>0, a<\delta / 6$; put $T(z)=z /(z+3 a)$. For $a>0$ and $\varepsilon>0$ we see via Lemma 2 that there is a $\xi>0$ and a $b>0$ satisfying

$$
\begin{gathered}
b<a, \\
\frac{-c}{-c+\xi}<\varepsilon \quad \text { where } c=\frac{-b}{-b+3 a}, \\
\text { if }-b \leqslant \operatorname{Re} z \leqslant 1 \text { and } \operatorname{Re}\{T(z)\} \leqslant \xi \text { then }|T(z)|<\varepsilon / 4 .
\end{gathered}
$$

Put $0<\beta<(\delta / Q)^{2}$ where $1 /(Q-1)<b$ and $Q \in \mathbf{Z}^{+}, Q>4$.

It follows from Proposition 1 that we can inductively choose $Q$ trigonometric polynomials $p_{1}, \ldots, p_{Q}$ such that

$$
\begin{gathered}
\left\|p_{i}\right\|_{\infty} \leqslant 1 \quad(i=1, \ldots, Q), \\
\int_{G} p_{i} d|\nu|>1-\beta^{2} \quad(i=1, \ldots, Q)
\end{gathered}
$$

and

$$
\bar{p}_{s} p_{t} \text { has frequencies in } \Gamma \backslash\{0\} \text { for } s<t \text {. }
$$

For each trigonometric polynomial $p_{i}$ define $E_{i}=\left\{x \in \Omega: \operatorname{Re} p_{i}(x)<1-\beta\right\}$. An easy calculation based on (1.5) gives $|\nu|\left(E_{i}\right)<\beta$ for each $i$. Put $E=\cup_{1}^{Q} E_{i}$; then $|\nu|(E)<\delta$. 
As in [2], we now define the trigonometric polynomial $g$ by

$$
g=\frac{2}{Q^{2}-Q} \sum_{s<t} \bar{p}_{s} p_{t}=u+i \tilde{v} .
$$

An easy computation using (1.4) shows that $\|g\|_{\infty}<1$ and $u>-1 /(Q-1)$; thus $-b<u<1$. A direct computation also establishes

$$
u(x)>1-4 \beta \quad(x \in \Omega \backslash E) .
$$

Write $g=g^{+}+g^{-}$where $g^{+}$is the analytic projection of $g$. Put $h=\left(g^{+}\right)+\overline{\left(g^{-}\right)}$; observe that $h$ has only positive frequencies. Unfortunately, although $\|g\|_{\infty}<1$, we cannot say as much of $h$. However, we will make much of the fact that $h$ and $g$ have the same real part.

Let $h=u+i v$; there is an $M \in \mathbf{Z}^{+}$such that $-M<v<M$ and thus by Lemma $1 T_{M}(h)=k$ is a trigonometric polynomial with positive frequencies such that $|T(h)-k|<\varepsilon / 4$,

$$
\|k\|_{\infty}^{2}<1+\delta
$$

and via (1.7)

$$
\operatorname{Re} k(x)>1-\delta \text { for all } x \in \Omega \backslash E .
$$

It is a direct consequence of (1.8) and (1.9) that $|1-k|<\varepsilon$ on $\Omega \backslash E$. Thus, (a) and (b) are now established.

We now wish to confirm (c). Put $k=u_{1}+i v_{1}$ and $F=\left\{x \in G: u_{1}>\xi\right\}$. Inasmuch as $k$ has only positive frequencies we see that $\int_{G} u_{1}(\theta) d \theta=0$. However, $c<u_{1}$ and $\int_{G} u_{1}(\theta) d \theta=0$ combine with (1.2) to give $m_{G}(F)<\varepsilon$. It follows from (1.3) and $|T(h)-k| \leqslant \varepsilon / 4$ that $|k|<\varepsilon$ on $\Omega \backslash F$. Our proof is complete.

Corollary 1 (Helson-Lowdenslager [6]). If $\Gamma$ is totally ordered and $\mu \in$ $M(G)$ is of analytic type so is $\mu_{s}$ and $\hat{\mu}_{s}(0)=0$.

Proof. Let $\mu \in M(G)$ be of analytic type; put $\mu=\mu_{a}+\mu_{s}$; we gather from Theorem 1 that there is a sequence $\left\langle k_{n}\right\rangle_{1}^{\infty}$ of trigonometric polynomials having only positive frequencies such that $k_{n} \rightarrow 1$ a.e. $\bmod \left|\mu_{s}\right|, k_{n} \rightarrow 0$ a.e. $\bmod m_{G}$ and $\left\|k_{n}\right\|_{\infty} \leqslant 2$. Notice that for all $\gamma \in \Gamma$

$$
\hat{\mu}_{s}(\gamma)=\int_{G}\left(1-k_{n}(x)\right) \gamma(-x) d \mu_{s}+\int_{G} k_{n}(x) \gamma(-x) d \mu_{s} .
$$

Fix $\gamma_{0} \leqslant 0$; it follows that

$$
\left|\hat{\mu}_{s}\left(\gamma_{0}\right)\right| \leqslant \int_{G}\left|1-k_{n}\right| d\left|\mu_{s}\right|+\left|\int_{G} k_{n}(x) \gamma_{0}(-x) d\left(\mu-\mu_{a}\right)\right| ;
$$

since $\mu$ is of analytic type, $k_{n}$ has only positive frequencies, and $\gamma_{0}<0$, we get

$$
\left|\hat{\mu}_{s}\left(\gamma_{0}\right)\right| \leqslant \int_{G}\left|1-k_{n}\right| d\left|\mu_{s}\right|+\int_{G}\left|k_{n}\right| d\left|\mu_{a}\right| .
$$

Well, let $n \rightarrow \infty$ in the above inequality; then both summands on the right side of the inequality converge to zero by the Lebesgue Dominated Convergence Theorem. Our proof is complete. 
It is not hard to see that if $\Gamma_{0} \subset \Gamma$ has finite index in $\Gamma$, we can also require in Theorem 1 that $k$ have frequencies in $\Gamma_{0} \backslash\{0\}$. With this remark in mind we obtain an infinite-dimensional version of a result of S. Bochner [1].

COROLlaRY 2. For any cardinal number $\omega$ let $\mathrm{T}^{\omega}$ denote the unrestricted product of $\omega$ copies of $\mathbf{T}$. Put $Q^{\omega}=\left\{\left\langle x_{\alpha}\right\rangle: x_{\alpha} \in \mathbf{Z}, x_{\alpha}\right\rangle 0$ for all $\left.\alpha\right\} \subset \hat{\mathbf{T}}^{\omega}$. If $\mu \in M\left(\mathbf{T}^{\omega}\right)$ and supp $\hat{\mu} \subset Q^{\omega}$ then $\mu \in M_{a}\left(T^{\omega}\right)$.

Proof. Suppose $\mu \in M\left(T^{\omega}\right)$ and supp $\hat{\mu} \subset Q^{\omega}$; fix $\xi_{0}=\left\langle\xi_{\alpha}\right\rangle \in \hat{\mathbf{T}}^{\omega}$ and choose $N>\left|\xi_{\alpha}\right|$ for all $\alpha\left(N \in \mathbf{Z}^{+}\right)$. Let $\Gamma_{0}^{*}$ be the group generated by $\xi_{0}$; there are a finite number of fixed indices $\alpha_{1}, \ldots, \alpha_{j}$ such that if $\left\langle y_{\alpha}\right\rangle \in \Gamma_{0}^{*}$ then $y_{\alpha}=0$ for all $\alpha \notin\left\{\alpha_{1}, \ldots, \alpha_{j}\right\}$. Let $\Gamma_{0}$ be the subgroup of $\hat{\mathbf{T}}^{\omega}$ such that if $\left\langle w_{\alpha}\right\rangle \in \Gamma_{0}$ then $w_{\alpha_{i}} \in N \mathbf{Z}$ for $i=1, \ldots, j$.

Well-order the indexing set for $\hat{\mathbf{T}}^{\boldsymbol{\omega}}$ and put $\boldsymbol{P}^{+}$equal to the positive cone (with respect to the usual lexicographic order) induced by the well-ordering. Since $\Gamma_{0}$ has finite index in $\hat{\mathbf{T}}^{\omega}$, there is a sequence of trigonometric polynomials $\left\langle\boldsymbol{k}_{n}\right\rangle_{1}^{\infty}$ having frequencies in $P^{+} \cap \Gamma_{0},\left\|k_{n}\right\|_{\infty} \leqslant 2$, such that $k_{n} \rightarrow 1$ a.e. $\bmod \left|\mu_{s}\right|$ and $k_{n} \rightarrow 0$ a.e. $\bmod m_{\mathrm{T}}$. Thus

$$
\left|\hat{\mu}_{s}\left(\xi_{0}\right)\right|<\int_{\mathbf{T}^{\omega}}\left|1-k_{n}\right| d\left|\mu_{s}\right|+\left|\int_{\mathbf{T}^{\omega}} k_{n}(x) \xi_{0}(-x) d\left(\mu-\mu_{a}\right)\right|
$$

since supp $\hat{\mu} \subset Q^{\omega}$ and Freq $k_{n} \subset P^{+} \cap \Gamma_{0}$, we obtain

$$
\begin{aligned}
\left|\hat{\mu}_{s}\left(\xi_{0}\right)\right| & \leqslant \int_{\mathbf{T}^{\omega}}\left|1-k_{n}\right| d\left|\mu_{s}\right|+\left|\int_{\mathbf{T}^{\omega}} k_{n}(x) \xi_{0}(-x) d \mu_{a}\right| \\
& \leqslant \int_{\mathbf{T}^{\omega}}\left|1-k_{n}\right| d\left|\mu_{s}\right|+\int_{\mathbf{T}^{\omega}}\left|k_{n}(x)\right| d\left|\mu_{a}\right| .
\end{aligned}
$$

Letting $n \rightarrow \infty$ yields $\hat{\mu}_{s}\left(\xi_{0}\right)=0$. Our proof is complete.

Perhaps Corollary 2 is new; in any event see [3, p. 191]. Theorem 1 can be placed in perspective by a reading of [5, pp. 43-44].

For discrete groups $\Gamma$, there is available another notion of order: If there exists a nontrivial homomorphism $\phi: \Gamma \rightarrow \mathbf{R}$, where $\mathbf{R}$ is the additive group of reals, we say $\Gamma$ is $\phi$-ordered and put $P=\phi^{-1}([0, \infty))$. Also let $P_{N}=\phi^{-1}([N, \infty)) ; \gamma$ is $\phi$-positive ( $\phi$-negative) if $\phi(\gamma)>0(\phi(\gamma)<0)$. Again, $\mu \in M(G)$ is said to be of analytic type if $\hat{\mu}(\gamma)=0$ for all $\gamma \in \Gamma \backslash P$. We point out that the obvious analogue of Theorem 1 is valid with the same proof if the kernel of $\phi$ is finite. Repeating the proof of Corollary 1 we obtain the following result: If $\operatorname{ker} \phi$ is finite and $\mu \in M(G)$ is of analytic type so is $\mu_{s}$ and $\hat{\mu}_{s}(\gamma)=0$ for all $\gamma \in \operatorname{ker} \phi$.

We say a subset $K \subset \Gamma$ is $\phi$-finite if there exists a finite set $B \subset \mathbf{R}$ such that $\phi(K) \subset B$. A subset $K \subset \Gamma$ is $\phi$-bounded if there exists a bounded set $B \subset \mathbf{R}$ such that $\phi(K) \subset B$.

We denote $(\operatorname{ker} \phi)^{\perp}$ by $G_{0} . M_{a G_{0}}(G)$ denotes the smallest $L$-ideal in $M(G)$ containing all measures of the form $m_{G_{0}} * \rho$ where $m_{G_{0}}$ is Haar measure on $G_{0}$ and $\rho \in M(G)$. Put $M_{s G_{0}}(G)=M_{a G_{0}}(G)^{\perp}=\{\nu \in M(G): \nu \perp \mu$ for each $\mu \in$ $\left.M_{a G_{0}}(G)\right\}$. For $\mu \in M(G)$ put $\mu=\mu_{a G_{0}}+\mu_{s G_{0}}$ where $\mu_{a G_{0}} \in M_{a G_{0}}(G)$ and $\mu_{s G_{0}} \in$ $M_{s G_{0}}(G)$. 
$\phi$ induces a continuous homomorphism $\phi^{*}: \mathbf{R} \rightarrow G$ given by $\left\langle\phi^{*}(x), \gamma\right\rangle=$ $e^{-i(x \phi(\gamma))}(x \in \mathbf{R}, \gamma \in \Gamma)$. Let $\Psi: M(\mathbf{R}) \rightarrow M(G)$ where $\Psi(\mu)(E)=\mu\left(\phi^{*-1}(E)\right)$ and $E$ is Borel in $G$. $M_{a \phi}(G)$ denotes the smallest $L$-ideal in $M(G)$ containing all measures of the form $\Psi(\omega) * \rho$ where $\omega \in M_{a}(\mathbf{R})$ and $\rho \in M(G)$. Put $M_{s \phi}(G)=$ $M_{a \phi}(G)^{\perp}=\left\{\nu \in M(G): \nu \perp \mu\right.$ for each $\left.\mu \in M_{a \phi}(G)\right\}$. For $\mu \in M(G)$ put $\mu=\mu_{a \phi}$ $+\mu_{s \phi}$ where $\mu_{a \phi} \in M_{a \phi}(G)$ and $\mu_{s \phi} \in M_{s \phi}(G)$. It is easy to see that $M_{a \phi}(G)$ coincides with the measures $\mu \in M(G)$ that translate continuously in the direction of $\phi$; i.e., $\lim _{x \rightarrow 0}\left\|\mu_{\phi^{*}(x)}-\mu\right\|=0$.

K. de Leeuw and I. Glicksberg proved that if $\Gamma$ is $\phi$-ordered and $\mu \in M(G)$ is of analytic type then $\mu \in M_{a \phi}(G)$; see [3]. An easy consequence of the de LeeuwGlicksberg result is: If $\mu \in M(G)$ is of analytic type and $\Gamma$ is $\phi$-ordered then $\mu_{s}$ is also of analytic type; see [3, p. 186]. We now show how to obtain these last two results with the methods of this paper.

Proposition 2. For $\mu \in M(G)$ and $K \phi$-finite in $\Gamma$

$$
\lim _{K \rightarrow \infty}\|\mu\|_{K}=\left\|\mu_{s G_{0}}\right\| \text {. }
$$

For $\mu \in M(G)$ and $K \phi$-bounded in $\Gamma$

$$
\lim _{K \rightarrow \infty}\|\mu\|_{K}=\left\|\mu_{s \phi}\right\| \text {. }
$$

Proof. The proof follows from easy modifications of [4]; see also [7].

THeOREM 2. Let $\Gamma$ be $\phi$-ordered. Let $\nu \in M_{s G_{0}}(G)$ and $\lambda \in M_{a G_{0}}(G)$, or let $\nu \in M_{s \phi}(G)$ and $\lambda \in M_{a \phi}(G)$. Let $\nu$ be concentrated on $\Omega_{\nu}$ and $\lambda$ be concentrated on $\Omega_{\lambda}$. Then given $\varepsilon>0$ there is a trigonometric polynomial $k$ having only $\phi$-positive frequencies, $E \subset \Omega_{\nu}, F \subset \Omega_{\lambda}$ such that

(a) $\|k\|_{\infty}<1+\varepsilon$

(b) $|k-1|<\varepsilon$ on $\Omega_{\nu} \backslash E$ and $|\nu|(E)<\varepsilon$;

(c) $|k|<\varepsilon$ on $\Omega_{\lambda} \backslash F$ and $|\lambda|(F)<\varepsilon$.

Furthermore, in the case $\nu \in M_{s \phi}(G)$ and $\lambda \in M_{a \phi}(G)$ we may choose $k$ to have frequencies in $P_{N}$ for any choice of $N$.

Proof. The proof is to interpret the argument of Theorem 1 in the $\phi$-ordered context.

First, suppose $\nu \in M_{s G_{0}}(G)$ and $\lambda \in M_{a G_{0}}(G)$. We may take $\lambda$ to be nonnegative. $\lambda \ll m_{G_{0}} * \lambda$. Thus suppose $\hat{\lambda}$ vanishes off $\operatorname{ker} \phi$ and in addition that $\|\lambda\|=1$. In the choice of the trigonometric polynomials $p_{1}, \ldots, p_{Q}$ replace (1.6) by

$$
\bar{p}_{s} p_{t} \text { has frequencies in } \Gamma \backslash \operatorname{ker} \phi \text { for } s<t \text {. }
$$

This can be done by Proposition 2. The remainder of the argument is repeated with $m_{G}$ replaced by $\lambda$. The crucial observation is that

$$
\int_{G} u_{1}(\theta) d \lambda(\theta)=0
$$

since $k$ has no frequencies in $\operatorname{ker} \phi$ and supp $\hat{\lambda} \subset \operatorname{ker} \phi$.

Now suppose $\nu \in M_{s \phi}(G)$ and $\lambda \in M_{a \phi}(G)$. Take $\lambda$ to be nonnegative. Let $f \in M(\mathbf{R}), f>0$, have compactly supported transform. Then $\lambda \ll \Psi(f) * \lambda$ and we 
may thus suppose $\hat{\lambda}$ has $\phi$-bounded support. Put $\|\lambda\|=1$ and fix $N$ in $Z^{+}$with supp $\hat{\lambda} \subset \Gamma \backslash P_{N}$. In the choice of the trigonometric polynomials $p_{1}, \ldots, p_{Q}$ replace (1.6) by

$$
\bar{p}_{s} p_{t} \text { has frequencies in } P_{N} \cup\left(-P_{N}\right) \text { for } s<t \text {. }
$$

This can be done by Proposition 2. The remainder of the argument is repeated with $m_{G}$ replaced by $\lambda$. The crucial observation is that

$$
\int_{G} u_{1}(\theta) d \lambda(\theta)=0
$$

since $k$ has its frequencies in $P_{N}$.

Corollary 3. Let $\mu \in M(G)$ be of analytic type for $\Gamma$ having a $\phi$-ordering. Then

(a) $\mu_{\phi s}=0$,

(b) $\hat{\mu}_{G_{0} s}(\gamma)=0$ for all $\gamma$ with $\phi(\gamma)<0$,

(c) $\hat{\mu}_{s}(\gamma)=0$ for all $\gamma$ with $\phi(\gamma)<0$.

Proof. Put $\mu=\mu_{\phi a}+\mu_{\phi s}$ and let $\gamma_{0} \in \Gamma$. Select from Theorem 2 a sequence $\left\langle k_{n}\right\rangle_{1}^{\infty}$ of trigonometric polynomials having frequencies in $P_{N}$ where $\left|\phi\left(\gamma_{0}\right)\right|<N$ such that $k_{n} \rightarrow 1$ a.e. $\bmod \left|\mu_{\phi s}\right|$ and $k_{n} \rightarrow 0$ a.e. $\bmod \left|\mu_{\phi a}\right|$. Then as in the proof of Corollary 1

$$
\left|\hat{\mu}_{\phi s}\left(\gamma_{0}\right)\right| \leqslant \int_{G}\left|1-k_{n}\right| d\left|\mu_{\phi s}\right|+\left|\int_{G} k_{n}(x) \gamma_{0}(-x) d\left(\mu-\mu_{\phi a}\right)\right| .
$$

But $k_{n} \bar{\gamma}_{0}$ has only $\phi$-positive frequencies since Freq $k_{n} \subset P_{N}$. So

$$
\left|\hat{\mu}_{\phi s}\left(\gamma_{0}\right)\right| \leqslant \int_{G}\left|1-k_{n}\right| d\left|\mu_{\phi s}\right|+\int_{G}\left|k_{n}\right| d\left|\mu_{\phi a}\right| .
$$

Both summands on the right converge to 0 as $n \rightarrow \infty$.

Now put $\mu=\left(=\mu_{\phi a}\right)=\mu_{G_{0} a}+\mu_{G_{0} \text { s. }}$. From Theorem 2 select a sequence $\left\langle k_{n}\right\rangle_{1}^{\infty}$ of trigonometric polynomials having $\phi$-positive frequencies such that $k_{n} \rightarrow 1$ a.e. $\bmod \left|\mu_{G_{0} s}\right|$ and $k_{n} \rightarrow 0$ a.e. $\bmod \left|\mu_{G_{0} a}\right|$ and $\left\|k_{n}\right\|_{\infty}<2$. Let $\gamma_{0} \in\{\gamma: \phi(\gamma)<0\}$. Then $\gamma_{0} k_{n}$ has only $\phi$-positive frequencies and the argument of the preceding paragraph repeats to give

$$
\hat{\mu}_{G_{0} s}=0 \text { on }\{\gamma: \phi(\gamma)<0\} .
$$

We now have $\mu_{G_{0} a}$ is analytic. Write $\mu_{G_{0} a}=\mu_{a}+\mu_{G_{0} a, s}$ where $\mu_{G_{0} a, s}$ is the singular part. It remains to see $\mu_{G_{0} a, s}$ is analytic. But $\mu_{G_{0} a, s} \ll m_{G_{0}} *\left|\mu_{G_{0} a, s}\right|=\nu$ is a positive singular measure, and therefore by Proposition 1 we may choose for any given finite set $F \subset \Gamma_{0}$ a sequence of trigonometric polynomials $\left\langle k_{n}\right\rangle_{1}^{\infty}$ such that Freq $k_{n} \subset \Gamma_{0} \backslash F, k_{n} \rightarrow 1$ a.e. $\bmod \left|\mu_{G_{0} a, s}\right|$ and $\left\|k_{n}\right\|_{\infty}<2$. Choose a trigonometric polynomial $s$ so that $\left\|s-\mu_{a}\right\|<\varepsilon$. Let $\gamma_{0}$ be $\phi$-negative. Determine $\left\langle k_{n}\right\rangle_{1}^{\infty}$ by the choice $F=\gamma_{0} \overline{(\text { Freq } s)} \cap \Gamma_{0}$. Now

$$
\begin{aligned}
\hat{\mu}_{\phi a, s}\left(\gamma_{0}\right) & =\int \bar{\gamma}_{0}\left(1-k_{n}\right) d \mu_{\phi a, s}+\int \bar{\gamma}_{0} k_{n} d \mu_{\phi a, s} \\
& \left.=\int \bar{\gamma}_{0}\left(1-k_{n}\right) d \mu_{\phi a, s}-\int \bar{\gamma}_{0} k_{n} d \mu_{a} \quad \text { (by the analyticity of } \mu_{\phi a}\right) .
\end{aligned}
$$


So $\left|\hat{\mu}_{\phi a, s}\left(\gamma_{0}\right)\right| \leqslant \int\left|1-k_{n}\right| d\left|\mu_{\phi a, s}\right|+\left|\int \bar{\gamma}_{0} k_{n} d \mu_{a}\right| . \int\left|1-k_{n}\right| d\left|\mu_{\phi a, s}\right| \rightarrow 0$ as $n \rightarrow \infty$. And $\left|\int \bar{\gamma}_{0} k_{n} d \mu_{a}\right| \leqslant\left|\int \bar{\gamma}_{0} k_{n} s d \theta\right|+2 \varepsilon=2 \varepsilon$ (since $\left\|\gamma_{0} k_{n}\right\|_{\infty}<2$ ).

\section{REFERENCES}

1. S. Bochner, Boundary values of analytic functions in several variables and of almost periodic functions, Ann. of Math. (2) 45 (1944), 708-722.

2. H. Davenport, On a theorem of P. J. Cohen, Mathematika 7 (1960), 93-97.

3. K. de Leeuw and I. Glicksberg, Quasi-invariance and analyticity of measures on compact groups, Acta Math. 109 (1963), 179-205.

4. R. Doss, On the transform of a singular or absolutely continuous measure, Proc. Amer. Math. Soc. 19 (1968), 361-363.

5. T. W. Gamelin, Uniform algebras, Prentice-Hall, Englewood Cliffs, N. J., 1969.

6. H. Helson and D. Lowdenslager, Prediction theory and Fourier series in several variables, Acta Math. 99 (1958), 165-201.

7. L. Pigno, Approximations to the norm of the singular part of a measure, Proc. Edinburgh Math. Soc. 20 (1976), 69-72.

Department of Mathematics, Kansas State University, Manhattan, Kansas 66506

Department of Mathematics, Illinois State University, Normal, IllinoIs 61761 